\title{
COMPOSICIÓN FLORÍSTICA DE MELASTOMATÁCEAS Y SU RELACIÓN CON EL ÁREA BASAL*
}

\author{
Nelly Llerena ${ }^{1}$, Roosevelt García ${ }^{2}$, Abel Monteagudo ${ }^{3}$, Cecilia Rodríguez ${ }^{2}$, Henrry Soplín ${ }^{4}$, Paola Tuesta $^{2}$ y \\ Kalle Ruokolainen
}

\begin{abstract}
RESUMEN
Estudiamos la composición de especies de melastomatáceas y su relación con el área basal de árboles en diferentes bosques de la Zona Reservada Allpahuayo - Mishana, Loreto, Perú, en el marco del curso "Uso de inventarios biológicos para revelar patrones de distribución geográfica de especies en la selva baja amazónica", organizado por el proyecto Diversidad Biológica de la Amazonía Peruana, Perú - Finlandia (BIODAMAZ), con el apoyo del Instituto de Investigaciones de la Amazonía Peruana (IIAP) y la Universidad Nacional de la Amazonía Peruana (UNAP). Reportamos 49 especies para el área, siendo Miconia el género más abundante, y con seis posibles nuevas especies. La mayor riqueza de especies estuvo representada por un bosque sobre suelo arcilloso, con 34 especies, que contrasta con un bosque sobre suelo arenoso de mal drenaje, con solo dos especies (Clidemia epibaterium y Tococa guianensis). No se encontró relación entre el área basal del bosque y la composición de melastomatáceas.
\end{abstract}

Palabras clave: Allpahuayo - Mishana, Amazonía peruana, área basal, índice de similitud, Melastomataceae, ordenación.

\begin{abstract}
We studied the species composition of Melastomataceae and its relation with basal area of trees in different forest types of Allpahuayo-Mishana Reserved Zone, Loreto, Peru, as part of the "Use of biological inventories to reveal geographic distribution patterns of lowland Amazonian species" course organized by the Peru-Finland Biological Diversity of the Peruvian Amazon project (BIODAMAZ), with the support of the Peruvian Amazonian Research Institute (IIAP) and the Peruvian Amazonian National University (UNAP). We report 49 species for the area, of which six may be new, and with Miconia as the most abundant genus. The greatest richness in species was found in forest on clay soil, with 34 species, contrasting with the poorly-drained forest on white sand that had only two species (Clidemia epibaterium and Tococa guianensis). There was no relation found between species composition of Melastomataceae and the basal area.
\end{abstract}

Key words: Allpahuayo-Mishana, basal area, Melastomataceae, ordenation, Peruvian Amazon, similarity index.

\footnotetext{
* Referencia bibliográfica del artículo: Llerena, N. García, R., Monteagudo, A., Rodríguez, C., Soplín, H., Tuesta, P. \& Ruokolainen, K. 2002. Composición florística de melastomatáceas y su relación con el área basal. En: Rodríguez, J.J., Ruokolainen, K. \& Soini, P. (eds.). La diversidad biológica de la Zona Reservada Allpahuayo - Mishana, Loreto, Perú: Relaciones entre distribución, abundancia y hábitats. Folia Amazónica 14 (1-2): NÚMEROS DE PÁGINA.

1 Universidad Nacional Mayor de San Marcos (UNMSM), Lima, Perú; correo electrónico: biodamaz@iiap.org.pe

2 Universidad Nacional de la Amazonía Peruana (UNAP), Iquitos, Perú.

3 Universidad Nacional San Antonio Abad del Cusco (UNSAAC), Cusco, Perú.

4 Universidad Nacional de San Martín (UNSM), Tarapoto, Perú.

5 Proyecto Diversidad Biológica de la Amazonía Peruana, Perú - Finlandia (BIODAMAZ) / Universidad de Turku, Turku, Finlandia.

1 Proyecto Diversidad Biológica de la Amazonía Peruana (BIODAMAZ) es un convenio entre los gobiernos del Perú y de Finlandia ejecutado conjuntamente por el Instituto de Investigaciones de la Amazonía Peruana (IIAP) y por el consorcio finlandés formado por la empresa de consultorías ambientales, Biota BD Oy, y por la Universidad de Turku.
} 


\section{INTRODUCCIÓN}

El conocimiento de la distribución geográfica de las especies es un componente importante para la comprensión de los procesos biológicos de la Amazonía (Tuomisto y Ruokolainen, 1997). Para manejar y conservar la biodiversidad de una manera inteligente y sostenible es urgente determinar áreas para la conservación en dicha región, ante el acelerado incremento de la deforestación (Fearnside, 1982; Skole y Tucker, 1993).

En términos prácticos, es imposible estudiar la distribución geográfica de todas las especies amazónicas en el campo, debido a los problemas logísticos que representa, la riqueza extrema de especies y la taxonomía no resuelta de su flora (Ruokolainen et al., 1997). Sin embargo, podemos concentrar los esfuerzos en determinados grupos de organismos -especies indicadoras-, que pueden darnos información suficiente de las características ambientales de los lugares donde ocurren otras especies (Ruokolainen et al., 1994; 1997; Tuomisto y Ruokolainen, 1998; Bach et al., 1999; Vormisto et al., 2000).

En ocho tipos de bosques en la Amazonía peruana se utilizó separadamente la composición de especies de melastomatáceas, pteridofitas y árboles, relacionándola con las características del suelo (Ruokolainen et al., 1997). Dado que estos grupos de plantas mostraron un patrón similar entre los distintos sitios, los resultados sugieren que tanto las pteridofitas como las melastomatáceas pueden ser usadas como indicadoras de tipos de bosque florísticamente diferentes, y pueden servir indirectamente como indicadores de la presencia de otros grupos de organismos.

Investigaciones realizadas en los bosques de Finlandia han mostrado que el área basal del bosque está relacionada con la presencia y/o abundancia de otras especies de plantas (K. Ruokolainen, com. pers.). Sin embargo, no tenemos información de que este mismo patrón de relación también se manifieste en los bosques tropicales. En esta investigación buscamos determinar si el área basal de un punto en el bosque está condicionando la composición de especies de Melastomataceae. Los resultados de este estudio pueden ayudarnos a mejorar nuestro conocimiento de cómo es la relación dentro de las comunidades vegetales en un bosque amazónico, con base en la composición de sus especies y el área basal.

El trabajo se realizó en el marco del curso "Uso de inventarios biológicos para revelar patrones de distribución geográfica de especies en la selva baja amazónica", organizado por el proyecto Diversidad Biológica de la Amazonía Peruana, Perú - Finlandia (BIODAMAZ) ${ }^{1}$, con el apoyo del Instituto de Investigaciones de la Amazonía Peruana (IIAP) y la Universidad Nacional de la Amazonía Peruana (UNAP).

\section{MATERIAL Y MÉTODO}

Realizamos el presente estudio en el mes de noviembre del 2000 en siete tipos de bosque ubicados en la Zona Reservada Allpahuayo - Mishana (ZRAM) y en la carretera Iquitos - Nauta. La ZRAM está ubicada en la Provincia de Maynas, Departamento de Loreto, entre el río Nanay y la carretera Iquitos - Nauta, y tiene una superficie de 57663.43 ha (UTM 9551657 N, 647589 E). En general, la ZRAM presenta bosques altos sobre suelo arcilloso relativamente rico de la Formación Pebas, bosques sobre suelo areno-arcilloso con diferentes gradientes de drenaje, y bosques sobre arena blanca profunda o delgada, llamados varillal o chamizal, respectivamente.

Realizamos un inventario de la composición de melastomatáceas en siete transectos de $200 \mathrm{~m}$ x $5 \mathrm{~m}$, divididos en ocho subunidades de $25 \mathrm{~m}$ x $5 \mathrm{~m}$ (Figura 1). Los nombres de los transectos (y sus tipos de suelo) fueron: 1 Pebas (suelo arcilloso), 2 Varillal Seco (suelo arenoso), 3 Terraza (suelo arcillo arenoso), 4 Supaychacra (suelo arcilloso probablemente inundado por una quebrada en raras ocasiones), 5 Varillal Húmedo (suelo arenoso), 6 Mitaya (suelo arcillo-arenoso), 7 Medicinal (suelo arcilloso) (Figura 1). 
a)

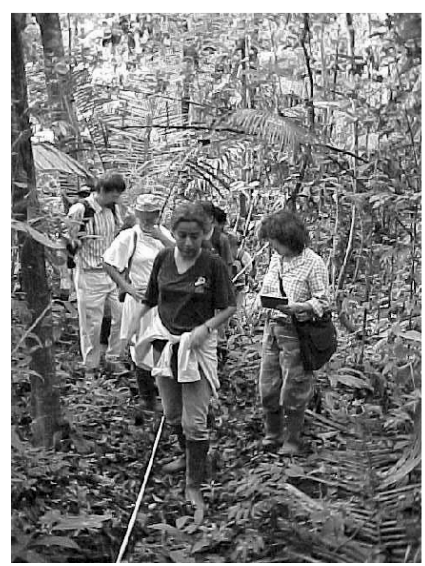

b)

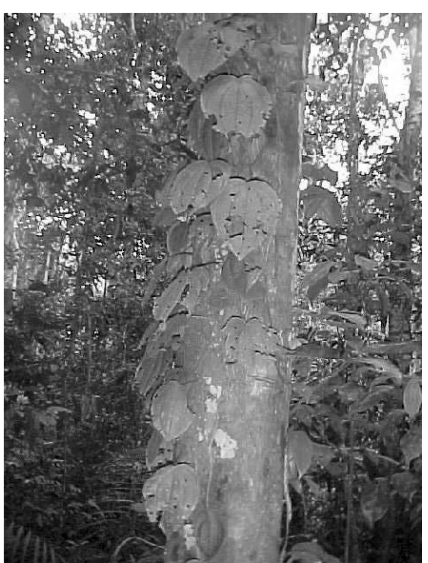

Figura 1. a) Establecimiento del transecto de 500 metros para la evaluación botánica, b) Clidemia epiphytica (Melastomataceae), una especie trepadora, encontrada principalmente en los hábitats con suelos arcillosos denominados aquí "Pebas" y "Supaychacra". Fotos: @IIAP-BIODAMAZ/J.J. Rodríguez.

Colectamos un ejemplar de todas las especies de melastomatáceas dentro de los transectos, y registramos el número de individuos por especie. Asimismo, colectamos individuos cuya identificación en el campo quedó incierta. Las muestras colectadas están depositadas en el Herbarium Amazonense (AMAZ) de la Universidad Nacional de la Amazonía Peruana (UNAP).

Medimos el área basal ( $\mathrm{m}^{2} / \mathrm{ha}$ ) en cuatro transectos de estudio (Supaychacra, Varillal Húmedo, Mitaya y Medicinal), en el centro de cada una de las ocho subunidades, mediante el método del relascopio. Este método consiste en utilizar una cinta métrica con una distancia ojo-brazo de $50 \mathrm{~cm}$. Contamos todos los árboles que a dicha distancia eran iguales o mayores al ancho del inicio de la cinta métrica $(1.9 \mathrm{~cm})$, (Pardé y Bouchon, 1994).

Para determinar los índices de similitud de composición florística de melastomatáceas entre transectos, utilizamos los indices de Steinhaus y Sørensen. El índice de Steinhaus considera la variación de las abundancias de las especies, mientras que el índice de Sørensen usa informacion de presencia y ausencia. Para hallar indices de similitud entre las áreas basales utilizamos el índice de distancia euclidiana, entre las subunidades de muestreo, dentro de los cuatro transectos de estudio. Para representar gráficamente nuestros resultados utilizamos el método de Ordenación de Coordenadas Principales. La correlación entre área basal y composición florística se calculó con la prueba de Mantel separadamente en los cuatro transectos de estudio, usando mil aleatorizaciones para estimar las probabilidades de error. Para facilitar la interpretación de las correlaciones los índices de Steinhaus y Sørensen fueron transformados a sus complementos, obteniendo así índices de distancia. Los análisis numéricos fueron hechos usando el paquete estadístico R-package.

\section{RESULTADOS}

Reportamos 49 especies de Melastomataceae con 2278 individuos en los siete transectos de estudio (7000 $\left.\mathrm{m}^{2}\right)$, (Anexo 1). El género con más especies fue Miconia (28 especies), que representa el 57\% de todas las especies encontradas. Las especies más abundantes en orden decreciente (número de individuos) fueron Clidemia epibaterium (606), Tococa guianensis (372), Miconia tetrasperma (233) y Leandra longicoma (168), que corresponden al $26.6 \%, 16.3 \%, 10.2 \%$ y $7.3 \%$, respectivamente, del total de especimenes colectados. La más alta riqueza de especies estuvo representada por el transecto Medicinal sobre suelo arcilloso, con 34 especies, que contrasta con el transecto Varillal Húmedo, con solo 2 especies (Tococa guianensis, Clidemia epibaterium), pero con una alta densidad de individuos sobre suelo arenoso (Figura 2). 
a)

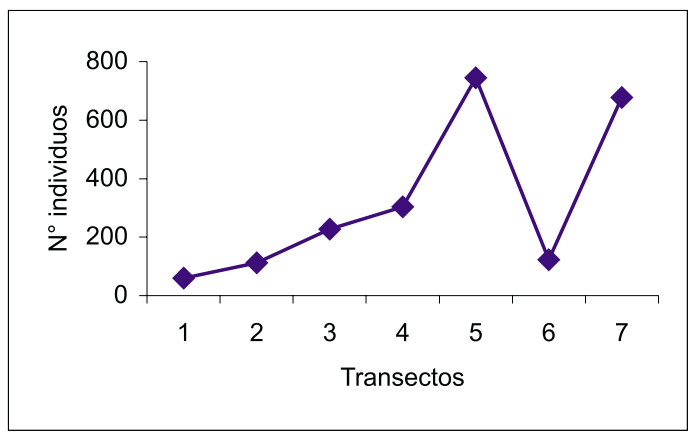

b)

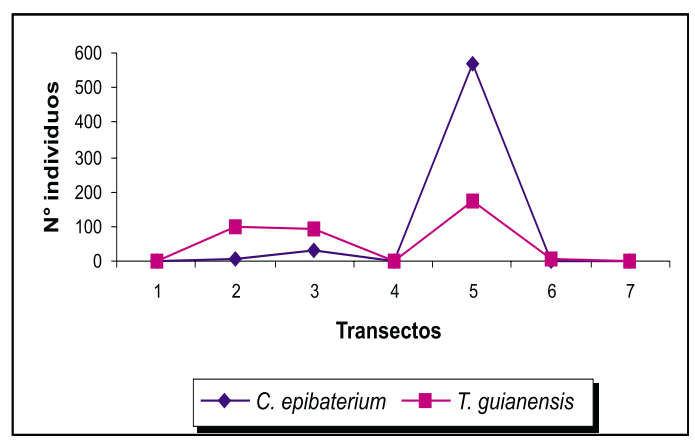

Figura 2. a) Número de individuos en todos los transectos evaluados, b) Número de individuos de Clidemia epibaterium y Tococa guianensis en los diferentes transectos evaluados. (1: Pebas, suelo arcilloso; 2:Varillal Seco, suelo arenoso; 3:Terraza, suelo arcillo-arenoso; 4:Supaychacra, suelo arcilloso probablemente inundable; 5:Varillal Húmedo, suelo arenoso; 6:Mitaya, suelo arcillo-arenoso; 7:Medicinal suelo arcilloso).

La relación entre el área basal y la composición florística de melastomatáceas y los sitios de muestreo, utilizando el índice de similitud de Steinhaus, muestra tres agrupamientos: 1) Varillal Seco, Terraza y Varillal Húmedo al lado izquierdo; 2) Supaychacra y Medicinal al lado superior derecho; 3) Mitaya y Pebas al lado inferior derecho (Figura 3a). Al ejecutar la ordenación con base en los índices de similitud de Sørensen (Figura 3b), el transecto denominado Terraza se separa de dos transectos denominados Varillales, el segundo grupo se mantiene prácticamente igual, y en el tercer grupo Pebas se distancia ligeramente de Mitaya.

a)

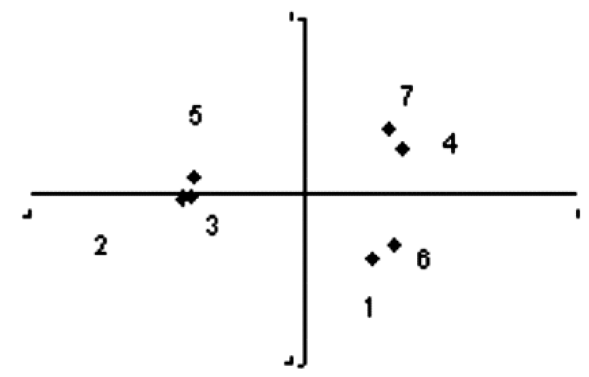

b)

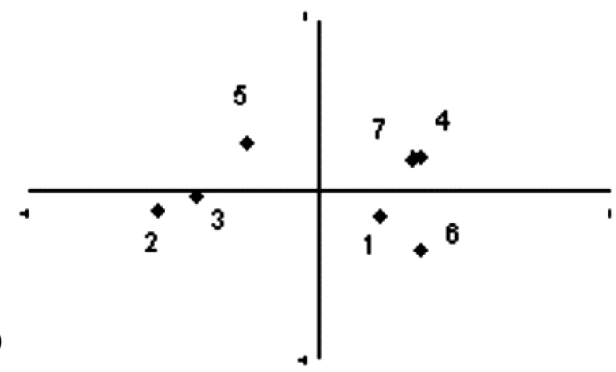

Figura 3. Agrupamiento de los transectos por el método de ordenación por coordenadas usando: a) el índice de similitud de Steinhaus (considera el número de individuos); b) el índice de similitud de Sørensen. En ambas ordenaciones se forman los mismos grupos: Grupo 1 (sup. der): Supaychacra y Medicinal; Grupo 2 (inf. der): Pebas y Mitaya; Grupo 3 (sup. izq y sup. der.) Varillal Seco, Terraza y Varillal Húmedo.

Luego de analizar la relación entre el área basal del bosque y la composición de especies de melastomatáceas en cada uno de los transectos, encontramos que no existe relación entre ambos. Los índices de similitud están débilmente correlacionados o están correlacionados negativamente (Tabla 1).

Tabla 1. Análisis de correlación de los transectos evaluados usando el método de Mantel.

\begin{tabular}{lcc}
\hline Transecto & Índice de correlación de Pearson $(\mathbf{r})$ & Probabilidad de error de $\mathbf{r}(\boldsymbol{\%})$ \\
\hline Supaychacra & -0.29 & 11.8 \\
Varillal Húmedo & 0.09 & 25.3 \\
Mitaya & 0.30 & 13.0 \\
Medicinal & -0.11 & 41.1 \\
\hline
\end{tabular}




\section{DISCUSIÓN}

El registro de 49 especies de melastomatáceas que reportamos en este trabajo de seis días de muestreo contrasta con el reporte de 31 especies de Vásquez (1997) para la Zona Reservada Allpahuayo - Mishana, en el cual incluye a las especies del género Mouriri, no consideradas en nuestra evaluación florística. Nuestro informe difiere en la composición de sus especies, a pesar de que el muestreo se ha realizado en la misma Zona Reservada, pero posiblemente en diferentes tipos de bosques (Anexo 1). Este informe incluye seis especimenes de Miconia que posiblemente corresponden a nuevas especies (K. Ruokolainen, com. pers.). La abundancia de especies de Miconia en esta área de estudio (28 spp., 57\% del total), no es sorprendente, pues es uno de los géneros de plantas neotropicales más grandes y representa el grueso de la composición de las melastomatáceas (Gentry, 1993).

A la escala local que trabajamos no encontramos evidencia de que exista alguna relación entre la composición de especies de melastomatáceas y el área basal del bosque. En caso de haberla, nuestros resultados pueden ser explicados por el tamaño reducido de la muestra, ya que es muy posible que las subunidades de los transectos ( 5 m x $25 \mathrm{~m}$ ) hayan sido insuficientes para lograr captar la posible variación entre ambos. Ruokolainen (1995), en un estudio sobre la variación florística y medioambiental en la Amazonía peruana, puntualizó que recién a partir de $100 \mathrm{~m}$ de transecto la muestra era suficientemente grande para poder captar la variación ambiental y florística. Es posible que trabajos a escalas más grandes puedan mostrarnos patrones que están ocultos por la escala local en que trabajamos, o bien nos demuestren que en realidad el área basal del bosque no está relacionada con la presencia de otros grupos de plantas en los bosques lluviosos de la Amazonía. Además de lo anterior, es posible que haya ocurrido un sesgo metodológico en nuestro estudio por haber ubicado los transectos a lo largo de trochas preestablecidas, lo que afecta la estimación del área basal y favorece la presencia y abundancia de especies que prefieren claros y zonas intervenidas (por ejemplo: Miconia amazonica, Miconia nervosa, Bellucia pentamera), lo que no nos habría permitido observar lo que sucede en un bosque en condiciones naturales. Sería conveniente que investigaciones posteriores consideraran realizar las evaluaciones en áreas poco intervenidas, alejadas de trochas principales, para evitar su posible influencia en los resultados.

La ubicación de los transectos de estudio según las coordenadas principales nos indica que hay tres agrupamientos: Grupo 1: Varillal Seco, Terraza y Varillal Húmedo; Grupo 2: Supaychacra y Medicinal; y Grupo 3: Mitaya y Pebas. Cuando se utilizan los resultados del índice de similitud de Steinhaus, que considera la abundancia de individuos, se ubica al transecto de Terraza junto a los dos bosques de tipo varillal, a pesar de tener características edáficas diferentes (Figura 3a), mientras que el índice de Sørensen hace que la distancia entre el transecto de Terraza y los varillales aumente, sobre todo con el Varillal Húmedo. Esto es debido posiblemente al índice en sí, que considera datos de presencia-ausencia, y por las distancias geográficas entre los transectos y diferencias en el drenaje del suelo. Pero, independientemente del índice utilizado, los transectos Varillal Seco y Terraza estuvieron geográficamente más cercanos entre sí que al Varillal Húmedo. Esto puede explicar su mayor semejanza en cuanto a la composición de sus especies compartidas y reflejadas en las Figuras $3 \mathrm{a}$ y $3 \mathrm{~b}$.

El transecto Varillal Húmedo presentó deficiencias en el drenaje con respecto a los otros transectos, lo que estaría condicionando qué plantas pueden estar presentes allí (Tabla 1). Este tipo de ambiente con condiciones especiales en los gradientes ambientales (tipo de suelo, drenaje, capa de materia orgánica), parece restringir la presencia de las especies de plantas (Anderson, 1981; Encarnación, 1985). En nuestro transecto de estudio sólo encontramos Clidemia epibaterium y Tococa guianensis. Así, el 94\% de individuos de C. epibaterium fue registrado en el Varillal Húmedo. T. guianensis parece tener un espectro más amplio de preferencias ambientales, pues, estuvo presente en cinco de los siete transectos estudiados, mientras que Maieta guianensis estuvo ausente en Varillal Húmedo (Anexo 1). Por causa de la extensión y complejidad de la Amazonía aún carecemos de mediciones de gradientes ambientales que nos permitan saber acerca de su influencia sobre estas especies, así como de las réplicas necesarias para reportar resultados concluyentes; es por ello que debemos seguir desarrollando metodologías rápidas y eficaces para su estudio. 


\section{AGRADECIMIENTOS}

Nuestro agradecimiento al proyecto BIODAMAZ, Perú - Finlandia, por la oportunidad de realizar este estudio.

\section{BIBLIOGRAFÍA}

ANDERSON, A.B. 1981. White sand vegetation of Brazilian Amazonia. Biotropica 13 (3): 199-210.

BACH, K.; KESSLER, M.; GONZALES, J. 1999. Caracterización preliminar de los bosques deciduos andinos en Bolivia en base a grupos indicadores botánicos. Ecología en Bolivia 32: 7-22.

ENCARNACIÓN, F. 1985. Introducción a la flora y vegetación de la Amazonía Peruana: estado actual de los estudios, medio natural y ensayo de claves de determinación de las formaciones vegetales en la llanura Amazónica. Candollea 40 (1): 237-252.

FEARNSIDE, P.M. 1982. Deforestation in the Brazilian Amazon: How fast is it occurring? Interciencia 7 (2): $82-85$

GENTRY, H.A. 1993. A field guide to the families and genera of wood plants of northwest South America (Colombia, Ecuador, Peru). Conservation International, Washington, D. C. 895 pp.

PARDÉ, J.; BOUCHON, J. 1994. Dasometria. 2da. Edición. Editorial Paraninfo. Madrid. España. 387 pp.

RUOKOLAINEN, K. 1995. Floristic and environmental variation in the rain forests of Peruvian Amazonia. Reports form the Department of Biology, University of Turku, Finland No. 50.

RUOKOLAINEN K.; LINNA, A.; TUOMISTO, H. 1997. Use of Melastomataceae and Pteridophytes for revealing phytogeographical patterns in Amazonian rain forests. Journal of Tropical Ecology 13: 243256.

RUOKOLAINEN, K.; TUOMISTO, H.; RÍOS, R.; TORRES, A.; GARCÍA, M. 1994. Comparación florística de doce parcelas en bosque de tierra firme en la Amazonía Peruana. Acta Amazónica 24 (1/2): 31-48.

SKOLE, D.; TUCKER, C. 1993. Tropical deforestation and habitat fragmentation in the Amazon: Satellite data from 1978 to 1988. Science 260: 1905-09.

TUOMISTO, H.; RUOKOLAINEN, K. 1997. The role of ecological knowledge in explaining biogeography and biodiversity in Amazonia. Biodiversity and Conservation 6: 347-357.

TUOMISTO, H.; RUOKOLAINEN, K. 1998. Uso de especies indicadoras para determinar características del bosque y de la tierra. En: Kalliola R.; Flores Paitán, S. (eds.). Geoecología y desarrollo amazónico: estudio integrado en la zona de Iquitos, Perú. Annales Universitatis Turkuensis Ser. A II 114: 481-491.

VÁSQUEZ, R. 1997. Flórula de las reservas biológicas de Iquitos, Perú. The Missouri Botanical Garden Press. EE.UU. 1046 pp.

VORMISTO, J.; PHILLIPS, O.L.; RUOKOLAINEN, K.; TUOMISTO, H.; VÁSQUEZ, R. 2000. A comparison of fine-scale distribution patterns of four plant groups in an Amazonian rainforest. Ecography 23: 349-359. 
Anexo 1. Especies y número de individuos de Melastomataceae en los siete transectos evaluados.

\begin{tabular}{|c|c|c|c|c|c|c|c|}
\hline ESPECIE & PEBAS & $\begin{array}{l}\text { VARIL. } \\
\text { SECO }\end{array}$ & TERRAZA & $\begin{array}{c}\text { SUPAY- } \\
\text { CHACRA }\end{array}$ & $\begin{array}{l}\text { VARIL. } \\
\text { HUMEDO }\end{array}$ & MITAYA & MEDICINAL \\
\hline Aciotis acuminifolia & 0 & 0 & 0 & 1 & 0 & 0 & 4 \\
\hline Adelobotrys boissieriana & 0 & 5 & 1 & 6 & 0 & 0 & 5 \\
\hline Adelobotrys macrantha & 0 & 0 & 0 & 0 & 0 & 0 & 2 \\
\hline Adelobotrys marginata & 0 & 0 & 17 & 0 & 0 & 0 & 0 \\
\hline Blackea rosea & 1 & 0 & 0 & 3 & 0 & 0 & 1 \\
\hline Clidemia allardii & 0 & 0 & 0 & 0 & 0 & 0 & 23 \\
\hline Clidemia epibaterium & 0 & 5 & 32 & 0 & 569 & 0 & 0 \\
\hline Clidemia epiphytica & 11 & 0 & 0 & 19 & 0 & 1 & 9 \\
\hline Leandra caquetana & 1 & 0 & 0 & 0 & 0 & 0 & 0 \\
\hline Leandra longicoma & 14 & 0 & 0 & 0 & 0 & 6 & 168 \\
\hline Leandra macdanielii & 0 & 0 & 7 & 0 & 0 & 0 & 8 \\
\hline Leandra secunda & 0 & 0 & 0 & 30 & 0 & 0 & 0 \\
\hline Loreya klugii & 0 & 0 & 0 & 0 & 0 & 0 & 1 \\
\hline Loreya umbellata & 0 & 0 & 8 & 0 & 0 & 0 & 0 \\
\hline Maieta guianensis & 16 & 4 & 9 & 26 & 0 & 22 & 17 \\
\hline Miconia tomentosa & 0 & 0 & 5 & 2 & 0 & 0 & 4 \\
\hline Miconia acutipetala & 1 & 0 & 0 & 1 & 0 & 2 & 35 \\
\hline Miconia aff. tetrasperma & 0 & 0 & 0 & 27 & 0 & 0 & 206 \\
\hline Miconia affinis & 0 & 0 & 0 & 0 & 0 & 0 & 1 \\
\hline Miconia amazonica & 0 & 0 & 0 & 0 & 0 & 0 & 10 \\
\hline Miconia ampla & 0 & 0 & 0 & 1 & 0 & 0 & 0 \\
\hline Miconia bubalina & 0 & 0 & 0 & 2 & 0 & 0 & 9 \\
\hline Miconia calvescens & 0 & 0 & 0 & 0 & 0 & 19 & 0 \\
\hline Miconia centrodesma & 4 & 0 & 0 & 0 & 0 & 0 & 5 \\
\hline Miconia duckei & 0 & 0 & 0 & 56 & 0 & 0 & 29 \\
\hline Miconia elata & 8 & 0 & 0 & 25 & 0 & 9 & 38 \\
\hline Miconia emandata & 0 & 0 & 0 & 30 & 0 & 0 & 0 \\
\hline Miconia grandifolia & 0 & 0 & 0 & 3 & 0 & 4 & 5 \\
\hline Miconia lugonis & 0 & 0 & 0 & 8 & 0 & 0 & 5 \\
\hline Miconia minutiflora & 0 & 0 & 0 & 1 & 0 & 0 & 3 \\
\hline Miconia napoana & 0 & 0 & 0 & 0 & 0 & 0 & 2 \\
\hline Miconia nervosa & 0 & 0 & 0 & 6 & 0 & 0 & 35 \\
\hline Miconia prassina & 0 & 0 & 0 & 0 & 0 & 0 & 4 \\
\hline Miconia pterocaulon & 0 & 0 & 19 & 0 & 0 & 0 & 0 \\
\hline Miconia punctata & 0 & 0 & 0 & 0 & 0 & 4 & 1 \\
\hline Miconia sp 63 & 0 & 0 & 0 & 0 & 0 & 0 & 1 \\
\hline Miconia sp. 53 & 0 & 0 & 0 & 0 & 0 & 3 & 0 \\
\hline Miconia sp. 54 & 0 & 0 & 0 & 0 & 0 & 2 & 0 \\
\hline Miconia sp. 61 & 0 & 0 & 0 & 0 & 0 & 15 & 5 \\
\hline Miconia sp. 8 & 4 & 0 & 0 & 0 & 0 & 0 & 12 \\
\hline Miconia sp.46 & 0 & 0 & 0 & 2 & 0 & 4 & 0 \\
\hline Miconia subspicata & 0 & 0 & 0 & 0 & 0 & 0 & 1 \\
\hline Miconia traillii & 0 & 0 & 1 & 0 & 0 & 0 & 0 \\
\hline Miconia umbriensis & 0 & 0 & 1 & 0 & 0 & 18 & 0 \\
\hline Ossaea boliviensis & 0 & 0 & 0 & 54 & 0 & 0 & 7 \\
\hline Tococa caquetana & 0 & 0 & 0 & 0 & 0 & 0 & 1 \\
\hline Tococa guianensis & 0 & 98 & 92 & 0 & 175 & 6 & 1 \\
\hline Tococa ulei & 0 & 0 & 35 & 0 & 0 & 8 & 19 \\
\hline Triolena amazonica & 0 & 0 & 0 & 0 & 0 & 32 & 0 \\
\hline
\end{tabular}

